\title{
Compact Galaxy Groups in the South
}

\author{
R. M. Price, B. Babic ${ }^{1}$ and K. Jones ${ }^{1}$
}

ATNF-CSIRO Australia and Department of Physics and Astronomy

\section{Introduction}

Compact groups are intermediate between single and binary galaxy systems, and clusters of galaxies. Because of their small numbers of members, and the small separations between the members of each group, they are good candidates for the study of galaxy interactions and mergers. In particular, high resolution imaging of these systems in neutral hydrogen can yield important data on interactions between galaxies.

The radio telescopes of the Australian National Telescope Facility (ATNF) are especially well suited to the study of neutral hydrogen gas in these systems. The Parkes 64-m telescope has a 15 arcminute FWHM beam at 21 centimeters wavelength which is sufficient to include the sky area of a typical compact group. Parkes was used for detection of $\mathrm{H}$ in nine southern groups and for determination of their approximate velocities, if not previously known. Followup observations with the ATNF Compact Array synthesis telescope provided high resolution (20 arcsecond) images with appropriate frequency resolution to determine velocity fields for the gas distribution.

Observations of $\mathrm{HI}$ in southern compact groups have also been reported by Williams and van Gorkom $(1985,1988)$, and Williams, McMahon, and van Gorkom (1991).

\section{The Observations}

We have carried out studies of nine compact galaxy groups in the southern skies, HCGs 22, 26, 42, 48, 63, 65, 91, AM1238-396, and ESO410-G. This is a preliminary report on these observations and some of the results The specific systems reported in this paper are HCG22 and HCG26 from the Hickson (1982) catalog of compact groups of galaxies. 1238-396 and ESO 410-G are reported by Babic et al., in this volume.

The Parkes observations were carried out over several periods in 1997-98. The $\mathrm{H}$ I receiver had a system temperature of $26 \mathrm{~K}$. The FWHM beamwidth was 15 arcminutes. A correlation spectrometer provided 1024 channels over $64 \mathrm{MHz}$, resulting in a final smoothed channel spacing of $15 \mathrm{~km} \mathrm{~s}^{-1}$. Observations were carried out using on/off techniques keeping the telescope at a constant altitude during adjacent scans. Observation times (on source) ranged from thirty minutes to four hours.

\footnotetext{
${ }^{1}$ Department of Physics, University of Queensland, Australia
} 
Follow-up observations with the ATNF Compact Array were carried out in 1998 using several configurations of the array to provide approximately 15 by 25 arcsecond resolution. Channel maps separated by either 15 or $20 \mathrm{~km} \mathrm{~s}^{-1}$ were produced. Data reduction was carried out using standard synthesis software along with additional routines produced by one of us (BB).

\section{HCG 22}

This is a group of three galaxies (classified as E2, Sa, and SBcd by Hickson, 1994) with concordant redshifts and within an area of five arcminutes. Only one of these galaxies shows any $\mathrm{H}$ I emission, galaxy (c) (using the nomenclature of Hickson, 1994), the SBcd. The H I distribution is remarkable in that it shows a lumpy torus with no signal detected in the central region of the galaxy at our level of sensitivity. The strongest concentration of $\mathrm{H} \mathrm{I}$ in the distribution is on the side toward the E2 galaxy, although there are no obvious tidal features. The hole in the center of the torus does not appear to align with the optical center of the galaxy, assuming the position is correct. The velocity distribution shows the spider pattern expected for an inclined spiral system, but perhaps affected by some departures from circular motion. None of the galaxies in this system is a continuum radio source at our level of sensitivity.

\section{HCG 26}

This is a dense group of seven galaxies within a three arcminute region. The group is dominated by a nearly edge-on system, galaxy (a), classified as Scd by Hickson (1994). As figure 1 shows, the region enveloping Hickson features (a), (b), (d), and (g) is easily detected and shows three discrete $\mathrm{HI}$ features. The preceeding, and strongest, is centered in the region of intersection of (a), (b), and (d). All three features seem to be extended N-S, but since this is the direction of elongation of the synthesized beam of the $\mathrm{CA}$, we cannot be certain of this. However, we note that in the region of the features (b) and (g) the digitized Sky Survey shows indications of faint optical structure also extending $\mathrm{N}-\mathrm{S}$. This could be evidence of debris from interactions between the galaxies. A small H I cloud is located about one arcminute below the following end of (a). It is not associated with any one of the identified members of the group. It might be a tidal feature or another result of an interaction. Confirmation of this suggestion will require further observations. We do not see the component of $\mathrm{H} \mathrm{I}$ extending down to galaxy (e), reported by Williams and van Gorkom (1992). Higher sensitivity and additional $u-v$ coverage may disclose this feature.

The velocity field for the three main features shows high latitude departures from circular motion. The velocity dispersion across the features is about 350 $\mathrm{km} \mathrm{s}^{-1}$. Complexity in the velocity field at each end of galaxy (a), and in some of the other smaller H I clouds seen in the vicinity, await further observations for a full interpretation.

There is an $8.5 \mathrm{mJy}$ continuum source seen at $12 \mathrm{~cm}$ wavelength, and located in the center of (a). Additionally, there is an indication of continuum radio emission from galaxy (e). The continuum emission in the region of galaxy (c) could be associated with that system, or be a background source. See figure 2 . 

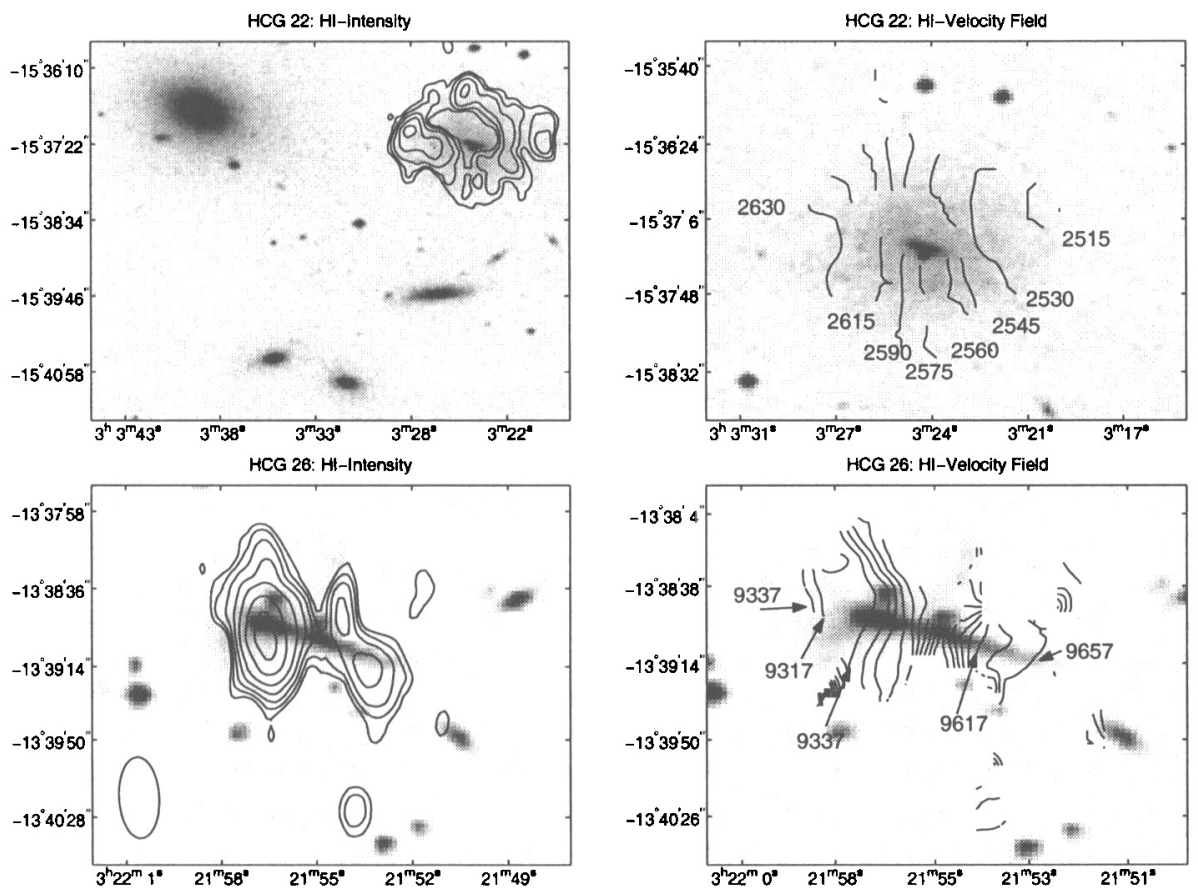

Figure 1. HCG 22 snd HCG 26: Integrated H I contours and HI velocity fields. For HCG 22 the contour levels are 25, 35, 50, 71, 90, and $100 \%$ of $0.3 \mathrm{Jy} /$ beam. Velocity contours are separated by $15 \mathrm{~km} \mathrm{~s}^{-1}$. For HCG 26 the contour levels are 12, 18, 25, 35, 50, 71, 90, 100\% of $0.5 \mathrm{Jy} /$ beam. Velocity contours are separated by $20 \mathrm{~km} \mathrm{~s}^{-1}$. 


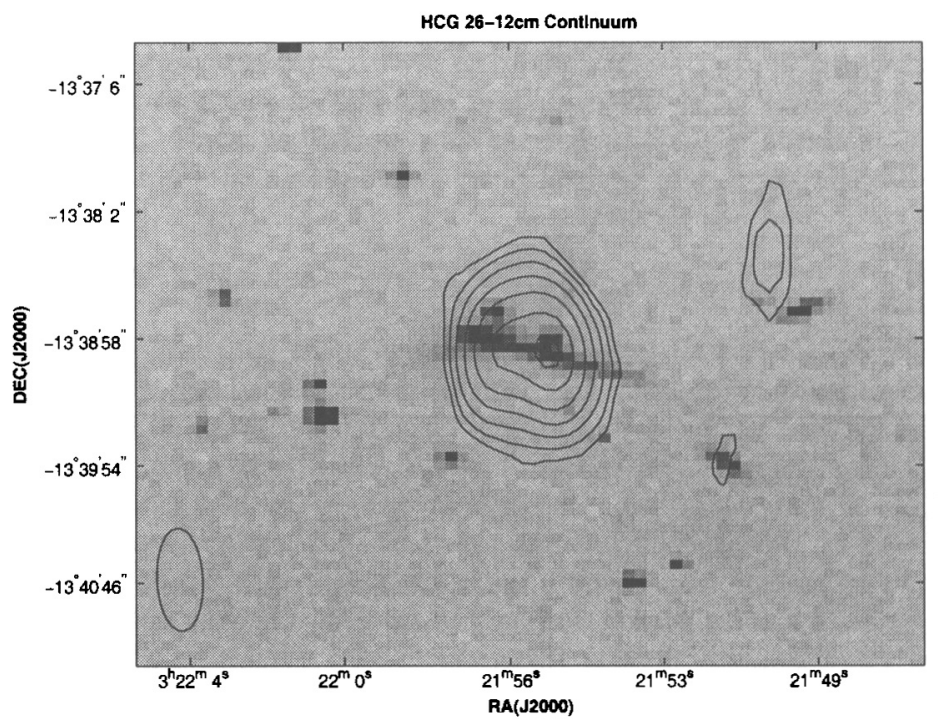

Figure 2. Continuum map of HCG 26 at 12 centimeter wavelength. Peak flux density of central source is $8.5 \mathrm{mJy}$.

\section{Remarks}

The $\mathrm{H}$ I distributions for these two groups fall into two of the catagories found for compact groups: $\mathrm{H}$ I associated only with the individual galaxies; a common $\mathrm{HI}$ envelope around two or more of the members of the group. With these systems, it appears that further observations with higher sensitivity and $u-v$ coverage will be required to identify clearly the features due to interactions, and to investigate the question of why these groups seem to be deficient in H I (Huchtmeier, 1997). We are continuing our studies of these and other southern compact groups.

\section{References}

Hickson, P. 1982, ApJ, 225, 382

Huchtmeier, W. 1997, A\&A, 325, 473

Williams, B. A. and van Gorkom, J. H. 1988, AJ, 95, 352

Williams, B. A., McMahon, P. M. and van Gorkom, J. H., 1991, AJ, 101, 1957

Williams, B. A. and van Gorkom, J. H. 1992, ASP Conference Series, 70, 77 